\title{
An examination of the effect of polarization on the radiation losses of bent optical fibres
}

\author{
A. ALTINTAS \\ Bilkent University, Department of Electrical and Electronics Engineering, \\ Bilkent, 06533 Ankara, Turkey \\ S. G. TANYER \\ Washington State University, School of Electrical Engineering and Computer \\ Science, Pullman, WA 99164, USA
}

Received 29 January; revised 26 August; accepted 16 September 1992

It has long been recognized that the bending losses in weakly guiding optical fibres are independent of the polarization for large bend radius. Here, we show this fact using the volume equivalent current method. The procedure is then applied to a helically bent fibre and it is shown that the radiation from the helical fibre is also independent of the polarization as long as the fibre is weakly guiding.

\section{Introduction}

It is well understood that a bent fibre radiates energy. It has been assumed that this radiation is independent of the polarization for large bend radius [1, 2]. This fact can be illustrated by the equivalent current method of Snyder and Love [2] as described in Section 3.

In the case of a helical fibre, the radiation is due to bending loss and helical loss [3]. If a multimode fibre is bent into a helix, the radiation acts as an effective cutoff for modes [4]. In the previous analyses, the polarization is assumed to stay parallel to a rectangular coordinate axis which is invariant with respect to the helical path. However, in reality, it is well known that the polarization slips back owing to the torsion of the helix [5]. Thus, one needs to include this rotation of polarization in the radiation calculations. We have performed this analysis and observed that the total radiation is independent of the polarization slip.

\section{Analysis for a circularly bent fibre}

The circularly bent fibre is shown in Fig. 1. The fibre has a core radius $\rho$, core refractive index $n_{\mathrm{co}}$ and cladding index $n_{\mathrm{cl}}$. The fibre and index parameters are denoted by $V$, and $\Delta$ as $V=k \rho\left(n_{\mathrm{co}}^{2}-n_{\mathrm{cl}}^{2}\right)^{1 / 2}, \Delta=\left(n_{\mathrm{co}}^{2}-n_{\mathrm{cl}}^{2}\right) / 2 n_{\mathrm{co}}^{2}$, where $k=2 \pi / \lambda_{0}$ and $\lambda_{0}$ is the free-space wavelength. $R_{\mathrm{c}}$ is the radius of curvature of the bend and it is assumed to be large 


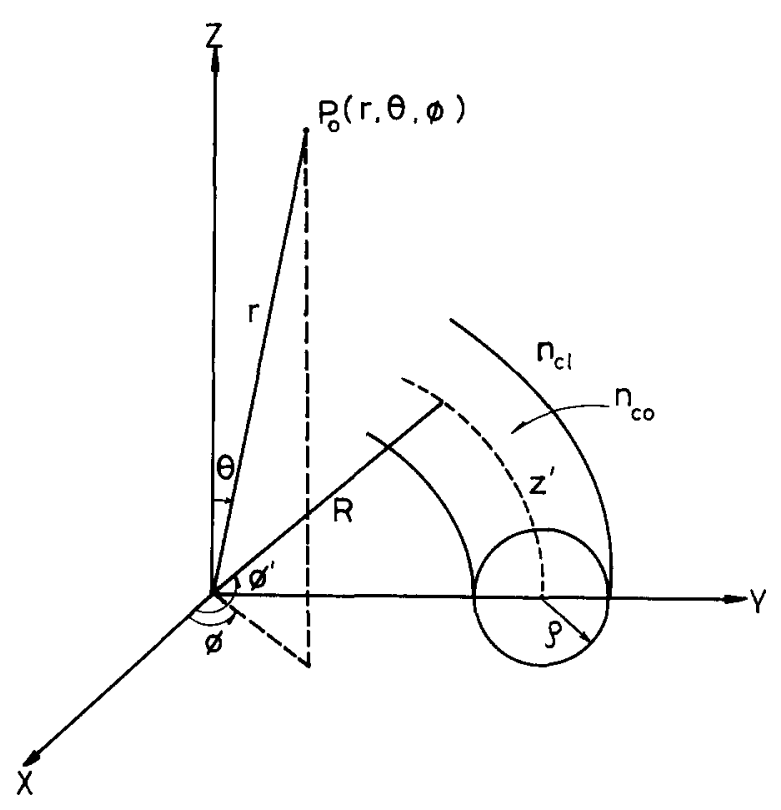

Figure 1 Circularly bent fibre.

compared to $\rho$ to satisfy the slowness criteria (equation 19-17 of [2]):

$$
\frac{R_{\mathrm{c}}}{\rho} \gg \frac{4 \pi}{\sqrt{2 \Delta}} \frac{V}{W^{2}}
$$

where $W=\rho\left(\beta^{2}-k^{2} n_{\mathrm{cl}}^{2}\right)^{1 / 2}$ and $\beta$ is the phase constant of the mode. As a typical example, for the following parameters:

$$
\begin{array}{rlrl}
n_{\mathrm{co}} & =1.560 & \lambda_{0} & =850 \mathrm{~nm} \\
n_{\mathrm{cl}} & =1.557 & \rho & =5 \mu \mathrm{m} \\
V & =3.574 & U & =1.857 \\
\Delta & =0.006 & W & =3.055
\end{array}
$$

the slowness criterion gives

$$
R_{\mathrm{c}} / \rho \gg 43.929
$$

which is satisfied for the bend radius in the range of a few millimetres.

The equivalent current method follows from Maxwell's equations. The core region of the bent fibre is replaced by an equivalent volume current of strength

$$
\mathbf{J}=i k\left(\frac{\epsilon_{0}}{\mu_{0}}\right)^{1 / 2}\left(n_{\mathrm{co}}^{2}-n_{\mathrm{cl}}^{2}\right) \mathbf{E}
$$

where $\mathbf{E}$ is the field in the core. If $\mathbf{E}$ is known or approximated then the radiation from the fibre is calculated by antenna theory. The easiest approximation, which is known to be valid when the slowness criteria is satisfied [2], is to take $\mathbf{E}$ as the mode in the core without any modifications. The radiation loss obtained by this method has been shown to agree with previous results [4]. 
In previous calculations, the core field is assumed to be linearly polarized in the direction perpendicular to the plane of the bend. Henceforth, this will be referred to as the 'perpendicular polarization' case. Here in this paper, we will be analysing the 'parallel polarization' case in which the core field is linearly polarized in the direction parallel to the plane of the bend (Fig. 1). The equivalent current in this parallel polarization case is given by

$$
\mathbf{J}=\mathrm{i}\left(\frac{\epsilon_{0}}{\mu_{0}}\right)^{1 / 2} k\left(n_{\mathrm{cl}}^{2}-n_{\mathrm{co}}^{2}\right) E \hat{\mathbf{a}}_{\mathrm{c}}
$$

where $\hat{\mathbf{a}}_{\mathrm{c}}$ is the unit vector along the radial direction away from the centre of curvature of the bend. For the single mode fibre, the electric field strength $E$ is given by

$$
E=\mathrm{e}^{i \beta z} F_{0}(R)
$$

with

$$
F_{0}(R)= \begin{cases}J_{0}(U R) / J_{0}(U) & \text { core } \\ K_{0}(W R) / K_{0}(W) & \text { cladding }\end{cases}
$$

where $J_{0}, K_{0}$ are Bessel functions of order $0, z$ is the distance along the fibre, $R=r / \rho$, and the parameter $U$ is given by

$$
U=\rho\left(\left(k n_{\mathrm{co}}\right)^{2}-\beta^{2}\right)^{1 / 2}
$$

Accordingly, the vector potential (equation 21-20 of [2]) is given as follows:

$$
\begin{aligned}
\mathbf{M}= & \int_{0}^{2 \pi} I_{\mathrm{c}} R_{\mathrm{c}} \exp \left\{\mathrm{i} R_{\mathrm{c}}\left[\beta \phi^{\prime}-k_{\mathrm{cl}} \sin \theta \cos \left(\phi-\phi^{\prime}\right)\right]\right\} \\
& \times\left[\hat{\mathbf{a}}_{\theta} \cos \theta \cos \left(\phi-\phi^{\prime}\right)+\hat{\mathbf{a}}_{\phi} \sin \left(\phi-\phi^{\prime}\right)\right] \mathrm{d} \phi^{\prime}
\end{aligned}
$$

where $I_{\mathrm{c}}=2 \pi \rho^{2} \int_{0}^{1} \mu R \mathrm{~d} R$, and $\mu=|\mathbf{J}|, k_{\mathrm{cl}}=k n_{\mathrm{cl}}$ and $z$ is approximated by $R_{\mathrm{c}} \phi^{\prime}$. In Equation 6 , only the transverse part which contributes to radiation is included. A careful examination of the above integral shows that the integrand oscillates quite rapidly except at stationary phase points which are given by

$$
\beta+k_{\mathrm{cl}} \sin \theta \sin \left(\phi-\phi^{\prime}\right)=0
$$

which yields

$$
\sin \left(\phi-\phi^{\prime}\right)=-\frac{\beta}{k_{\mathrm{cl}} \sin \theta}
$$

Using these values, the integral in Equation 6 is well approximated by

$$
\mathbf{M}=\left(\hat{\mathbf{a}}_{\theta} \cos \theta \sqrt{1-\left(\frac{\beta}{k_{\mathrm{cl}} \sin \theta}\right)^{2}}+\hat{\mathbf{a}}_{\phi} \frac{-\beta}{k_{\mathrm{cl}} \sin \theta}\right) 2 \pi R_{\mathrm{c}} I_{\mathrm{c}} \cdot\left|J_{\nu}\left(k_{\mathrm{cl}} R_{\mathrm{c}} \sin \theta\right)\right|
$$

where $\nu=\beta R_{\mathrm{c}} \gg 1$. The total radiated power in the far zone is given by

$$
P_{\mathrm{rad}}=\sigma \int_{0}^{2 \pi} \int_{0}^{\pi}\left[\left|M_{\theta}\right|^{2}+\left|M_{\phi}\right|^{2}\right] \sin \theta \mathrm{d} \theta \mathrm{d} \phi
$$

where $\sigma=\left(k^{2} n_{\mathrm{cl}} / 32 \pi^{2}\right)\left(\mu_{0} / \epsilon_{0}\right)^{1 / 2}$. Substituting Equation 9 into Equation 10 and setting 
$n_{\mathrm{co}} \cong n_{\mathrm{cl}}$, we obtain

$$
P_{\mathrm{rad}}=\frac{\pi}{8} \frac{R_{\mathrm{c}}^{2}}{\rho^{2}}\left(\frac{\mu_{0}}{\epsilon_{0}}\right)^{1 / 2} \frac{V^{2}}{n_{\mathrm{co}}} \frac{I_{\mathrm{c}}^{2}}{\Delta} \int_{0}^{\pi} J_{\nu}^{2}\left(k R_{\mathrm{c}} n_{\mathrm{cl}} \sin \theta\right)\left(f_{1}^{2}(\theta)+f_{2}^{2}(\theta)\right) \mathrm{d} \theta
$$

where

$$
\begin{aligned}
& f_{1}(\theta)=\cos \theta \sqrt{1-\left(\frac{\beta}{k_{\mathrm{cl}} \sin \theta}\right)^{2}} \\
& f_{2}(\theta)=\frac{\beta}{k_{\mathrm{cl}} \sin \theta}
\end{aligned}
$$

If we use Debye's approximate value for the Bessel function in the integrand, we get

$$
P_{\mathrm{rad}}=\frac{1}{8} \frac{R_{\mathrm{c}}}{\rho^{2}}\left(\frac{\mu_{0}}{\epsilon_{0}}\right)^{1 / 2} \frac{V^{2}}{n_{\mathrm{co}}} \frac{I_{\mathrm{c}}^{2}}{\Delta} \int_{0}^{\pi} \frac{\sin \theta Q(\theta)}{\left(\beta^{2}-k_{\mathrm{cl}}^{2} \sin ^{2} \theta\right)^{1 / 2}} \mathrm{e}^{S(\theta)} \mathrm{d} \theta
$$

where

$$
S(\theta)=-\frac{2}{3} \frac{k_{\mathrm{cl}} R_{\mathrm{c}}}{\sin ^{2} \theta}\left(\frac{\beta^{2}}{k_{\mathrm{cl}}^{2}}-\sin ^{2} \theta\right)^{3 / 2}
$$

and

$$
Q(\theta)=f_{1}^{2}(\theta)+f_{2}^{2}(\theta)
$$

Now, we can search for a stationary phase point of the above integral.

$$
\begin{aligned}
\frac{\partial S(\theta)}{\partial \theta} & =0 \\
& \Rightarrow\left(\frac{\beta^{2}}{k_{\mathrm{cl}}^{2}}-\sin ^{2} \theta\right)^{1 / 2}\left(-\frac{3}{2} \sin ^{2} \theta-\frac{\beta^{2}}{k_{\mathrm{cl}}^{2}}-\sin ^{2} \theta\right)=0 \\
& \Rightarrow \sin ^{2} \theta=\frac{\beta^{2}}{k_{\mathrm{cl}}^{2}} \cong 1
\end{aligned}
$$

Therefore the stationary phase point is very close to $\pi / 2$, so that $\theta=\pi / 2$ can be substituted into the coefficient function of the Bessel function in the integrand. Then we get

$$
P_{\mathrm{rad}}=\frac{\sqrt{\pi}}{32} I_{\mathrm{c}}^{2}\left(\frac{R_{\mathrm{c}}}{\rho}\right)^{1 / 2}\left(\frac{\mu_{0}}{\epsilon_{0}}\right)^{1 / 2} \frac{V^{2}}{W^{3 / 2}} \frac{n_{\mathrm{co}}}{\Delta} \exp \left(-\frac{4}{3} \frac{R_{\mathrm{c}}}{\rho} \frac{\Delta W^{3}}{V^{2}}\right)
$$

which is the same result as in the perpendicular polarization case [2]. It is also noted that, in the asymptotic evaluation of Equation 11, the contribution from $M_{\theta}$ is zero (since $f_{1}(\theta)=0$ at $\theta=\pi / 2$ ). For the perpendicular polarization case the contribution from $M_{\phi}$ is zero. The radiated fields of both polarizations are orthogonal, so the power loss is additive for the parallel and perpendicular components of an arbitrarily polarized field. 


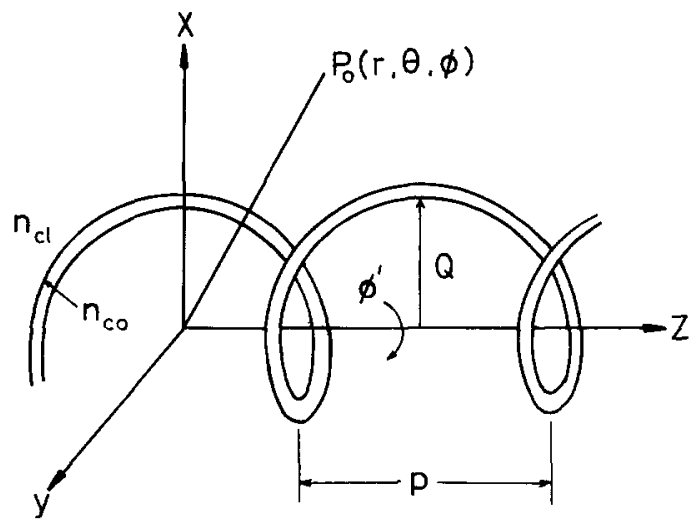

Figure 2 Helically bent fibre

\section{Helically bent single-mode fibre}

A helical fibre is shown in Fig. 2. The helix has a pitch $p$ and offset $Q$. The helix angle $\theta_{p}$ is defined by $\cos \theta_{p}=p /\left(p^{2}+(2 \pi Q)^{2}\right)^{1 / 2}$. The helix axis coincides with the $z^{\prime}$-axis and $\theta$ and $\phi$ are the spherical angles as shown in Fig. 2.

It has been shown that the direction of polarization of the field rotates along the helical path due to the torsion of the helix [5]. Let $\alpha$ denote the speed of this rotation, then the equivalent current is given by

$$
\mathbf{J}_{\mathrm{eq}}=\left[\hat{\mathbf{a}}_{x} \cos \alpha \phi^{\prime}+\hat{\mathbf{a}}_{y} \sin \alpha \phi^{\prime}\right] \mu
$$

in the core region, with $\mu$ being the magnitude in Equation 1. The vector potential becomes

$$
\mathbf{M}=\hat{\mathbf{a}}_{x} M_{x}+\hat{\mathbf{a}}_{y} M_{y}
$$

where

$$
\begin{aligned}
M_{\mathrm{r}}= & \mu \int_{-L}^{L} \exp \left[-j\left(\frac{\beta}{\cos \theta_{p}}-k_{\mathrm{cl}} \cos \theta\right) z^{\prime}\right. \\
& \left.-j k_{\mathrm{cl}} Q \sin \theta \cos \left(\phi-\frac{2 \pi}{p} z^{\prime}\right)\right]\left\{\begin{array}{c}
\cos \left(\alpha \frac{2 \pi}{p} z^{\prime}\right) \\
\sin \left(\alpha \frac{2 \pi}{p} z^{\prime}\right)
\end{array}\right\} \mathrm{d} z^{\prime}
\end{aligned}
$$

To get the radiation from a helix of infinite length, $L$ must be taken to infinity. Substituting $M_{\phi}=-M_{x} \sin \phi+M_{y} \cos \phi$ and $M_{\theta}=M_{x} \cos \phi \cos \theta+M_{y} \sin \phi \sin \theta$, we obtain

$$
\begin{aligned}
P_{\text {rad }}= & \sigma \int_{0}^{\pi} \int_{0}^{2 \pi}\left[\left|M_{r}\right|^{2}\left(\sin ^{2} \phi+\cos ^{2} \phi \cos ^{2} \theta\right)\right. \\
& +\left|M_{y}\right|^{2}\left(\cos ^{2} \phi+\cos ^{2} \theta \sin ^{2} \phi\right) \\
& \left.+\left|M_{x}\right|\left|M_{y}\right| \sin \phi \cos \phi\left(\cos ^{2} \theta-1\right)\right] \sin \theta \mathrm{d} \theta \mathrm{d} \phi
\end{aligned}
$$

It is also noted that the last term in the integrand vanishes after the $\phi$-integration. Using 
the following decompositions,

$$
\begin{aligned}
& \cos \left(\alpha \frac{2 \pi}{p} z^{\prime}\right)=\frac{1}{2}\left[\exp \left(j \alpha \frac{2 \pi}{p} z^{\prime}\right)+\exp \left(-j \alpha \frac{2 \pi}{p} z^{\prime}\right)\right] \\
& \sin \left(\alpha \frac{2 \pi}{p} z^{\prime}\right)=\frac{1}{2}\left[\exp \left(j \alpha \frac{2 \pi}{p} z^{\prime}\right)-\exp \left(-j \alpha \frac{2 \pi}{p} z^{\prime}\right)\right]
\end{aligned}
$$

Equation 21 can be written as

$$
\begin{aligned}
& M_{x}=M_{x a}+M_{x b} \\
& M_{y}=M_{y a}-M_{y b}
\end{aligned}
$$

where

$$
\begin{aligned}
M_{x_{b}^{a}}= & \frac{\mu}{2} \int_{-L}^{L} \exp \left[-j\left(\frac{\beta}{\cos \theta_{p}}-k_{\mathrm{cl}} \cos \theta \mp \alpha \frac{2 \pi}{p}\right) z^{\prime}\right] \\
& \times \exp \left[-j k_{\mathrm{cl}} Q \sin \theta \cos \left(\phi-\frac{2 \pi}{p} z^{\prime}\right)\right] \mathrm{d} z^{\prime}
\end{aligned}
$$

and

$$
\begin{aligned}
M_{y_{b}^{a}}= & \frac{\mu}{2 j} \int_{-L}^{L} \exp \left[-j\left(\frac{\beta}{\cos \theta_{p}}-k_{\mathrm{cl}} \cos \theta \mp \alpha \frac{2 \pi}{p}\right) z^{\prime}\right] \\
& \times \exp \left[-j k_{\mathrm{cl}} Q \sin \theta \cos \left(\phi-\frac{2 \pi}{p} z^{\prime}\right)\right] \mathrm{d} z^{\prime}
\end{aligned}
$$

The radiated power expression can be written as

$$
\begin{aligned}
P_{\mathrm{rad}}= & \sigma \int_{0}^{\pi} \int_{0}^{2 \pi}\left[\left|M_{x a}+M_{x b}\right|^{2}\left(\sin ^{2} \phi+\cos ^{2} \phi \cos ^{2} \theta\right)\right. \\
& \left.+\left|M_{y a}-M_{y b}\right|^{2}\left(\cos ^{2} \phi+\cos ^{2} \theta \sin ^{2} \phi\right)\right] \sin \theta \mathrm{d} \theta \mathrm{d} \phi
\end{aligned}
$$

Using the relation

$$
\exp (-j z \cos \theta)=J_{0}(z)+2 \sum_{m=1}^{\infty}(j)^{m} J_{m}(z) \cos (m \theta)
$$

we have

$$
\begin{aligned}
& M_{x_{b}^{a}}=\frac{\mu}{2}\left[J_{0}\left(k_{\mathrm{cl}} Q \sin \theta\right) F_{0_{b}^{a}}+2 \sum_{m=1}^{\infty}(j)^{m} J_{m}\left(k_{\mathrm{cl}} Q \sin \theta\right) F_{m_{b}^{a}}\right] \\
& M_{y_{h}^{a}}=\frac{\mu}{2 j}\left[J_{0}\left(k_{\mathrm{cl}} Q \sin \theta\right) F_{0_{b}^{a}}+2 \sum_{m=1}^{\infty}(j)^{m} J_{m}\left(k_{\mathrm{cl}} Q \sin \theta\right) F_{m_{b}^{a}}\right]
\end{aligned}
$$

where

$$
F_{m_{b}^{a}}=\exp \left\{j m \phi \frac{\sin \left[L\left(\frac{\beta}{\cos \theta_{p}}-k_{\mathrm{cl}} \cos \theta \pm \frac{\alpha 2 \pi}{p}+\frac{2 m \pi}{p}\right)\right]}{L\left(\frac{\beta}{\cos \theta_{p}}-k_{\mathrm{cl}} \cos \theta \pm \frac{\alpha 2 \pi}{p}+\frac{2 m \pi}{p}\right)}\right\}
$$




$$
+\exp \left\{-j m \phi \frac{\sin \left[L\left(\frac{\beta}{\cos \theta_{p}}-k_{\mathrm{cl}} \cos \theta \pm \frac{\alpha 2 \pi}{p}-\frac{2 m \pi}{p}\right)\right]}{L\left(\frac{\beta}{\cos \theta_{p}}-k_{\mathrm{cl}} \cos \theta \pm \frac{\alpha 2 \pi}{p}-\frac{2 m \pi}{p}\right)}\right\}
$$

Each term has a $(\sin x) / x$ behaviour which has a maximum at those points where the denominator vanishes and it is essentially zero everywhere else, since we are interested in the limit $L \rightarrow \infty$. Therefore, the doubly infinite set of terms in $\left|M_{x_{b}^{a}}\right|^{2}$ and $\left|M_{y_{b}^{a}}\right|^{2}$ simplify to the following:

$$
\begin{aligned}
\left|M_{x_{b}^{a}}\right|^{2}= & \left|M_{y_{b}^{a}}\right|^{2}=\frac{\mu^{2}}{4} L^{2} J_{0}^{2}\left(k_{\mathrm{c}} Q \sin \theta\right)\left[\frac{\sin ^{2}\left[A_{b}^{a}\right]}{\left[A_{b}\right]^{2}}\right] \\
& +\mu^{2} L^{2} \sum_{m=1}^{\infty} J_{m}^{2}\left(k_{\mathrm{cl}} Q \sin \theta\right)\left[\frac{\sin ^{2}\left[B_{b}\right]}{\left[B_{b}\right]^{2}}+\frac{\sin ^{2}\left[C_{b}\right]}{\left[C_{b}\right]^{2}}\right]
\end{aligned}
$$

where

$$
\begin{aligned}
A_{b}^{a} & =L\left(\frac{\beta}{\cos \theta_{p}}-k_{\mathrm{cl}} \cos \theta \mp \alpha \frac{2 \pi}{p}\right) \\
B_{b} & =L\left(\frac{\beta}{\cos \theta_{p}}-k_{\mathrm{cl}} \cos \theta \mp \alpha \frac{2 \pi}{p}+\frac{2 m \pi}{p}\right) \\
C_{b} & =L\left(\frac{\beta}{\cos \theta_{p}}-k_{\mathrm{cl}} \cos \theta \mp \alpha \frac{2 \pi}{p}-\frac{2 m \pi}{p}\right)
\end{aligned}
$$

The cross terms in $\left|M_{x}\right|^{2}$ and $\left|M_{y}\right|^{2}$ contain $M_{x a} M_{x b}^{*}$ or $M_{y a} M_{y b}^{*}$, respectively (the superscript $\left(^{*}\right)$ denotes complex conjugation). These terms are equal in magnitude and their total contribution to the integral in Equation 29 is zero. Since the speed $\alpha$ is given by

$$
\alpha=\cos \left(\theta_{p}\right)
$$

for vanishingly small fibre thickness compared with the radius of the curvature, some of the $(\sin x) / x$ functions will have peaks at imaginary values of $\theta$. The contribution of these peaks at imaginary values of $\theta$ has to be discarded for the power loss calculation (as in array antennas), and $\left|M_{x_{h}^{a}}\right|^{2}$ is written as

$$
\begin{aligned}
\left|M_{x_{b}^{a}}\right|^{2}= & \frac{\mu^{2}}{4} L^{2} J_{0}^{2}\left(k_{\mathrm{cl}} Q \sin \theta\right)\left[\frac{\sin ^{2}\left[A_{b}\right]}{\left[A_{b}\right]^{2}}\right] \\
& +\mu^{2} L^{2} \sum_{m=m_{\min }}^{m=m_{\max }} J_{m}^{2}\left(k_{\mathrm{cl}} Q \sin \theta\right)\left[\frac{\sin ^{2}\left[B_{b}\right]}{\left[B_{b}\right]^{2}}+\frac{\sin ^{2}\left[C_{b}\right]}{\left[C_{b}\right]^{2}}\right]
\end{aligned}
$$

where $m$ are values for which

$$
\cos \theta_{m}=\frac{\beta}{k_{\mathrm{cl}} \cos \theta_{p}} \mp \frac{a 2 \pi}{p k_{\mathrm{cl}}}-\frac{2 m \pi}{p k_{\mathrm{cl}}}
$$

yields a value of $\theta_{m}$ between 0 and $\pi$. The expression for $\left|M_{y_{h}^{a}}\right|^{2}$ is exactly the same, in other words,

$$
\left|M_{x_{b}^{a}}\right|^{2}=\left|M_{y_{b}^{a}}\right|^{2}=\left|M_{b}\right|^{2}
$$


Substituting these in Equation 29, and noting that $\left|M_{b}^{a}\right|^{2}$ is independent of $\phi$, we get

$$
P_{\mathrm{rad}}=2 \pi \sigma \int_{0}^{\pi}\left(\left|M_{a}\right|^{2}+\left|M_{b}\right|^{2}\right)\left(1+\cos ^{2} \theta\right) \sin \theta \mathrm{d} \theta
$$

Defining

$$
\begin{aligned}
q_{\mp} & =L\left(\frac{\beta}{\cos \theta_{p}}-k_{\mathrm{cl}} \cos \theta \mp \frac{\alpha 2 \pi}{p}-\frac{2 m \pi}{p}\right) \\
d q_{\mp} & =L k_{\mathrm{cl}} \sin \theta \mathrm{d} \theta
\end{aligned}
$$

the integral in Equation 42 becomes

$$
\begin{aligned}
P_{\mathrm{rad}}= & 2 \pi \sigma \mu^{2} L^{2} \sum_{m} J_{m}^{2}\left(k_{\mathrm{cl}} Q \sin \theta_{m}\right)\left(1+\cos ^{2} \theta_{m}\right) \frac{1}{L k_{\mathrm{cl}}} \\
& \times\left[\int_{z^{-}}^{z^{+}} \frac{\sin ^{2}\left(q_{+}\right)}{q_{+}^{2}} \mathrm{~d} q_{+}+\int_{z^{-}}^{z^{+}} \frac{\sin ^{2}\left(q_{-}\right)}{q_{-}^{2}} \mathrm{~d} q_{-}\right]
\end{aligned}
$$

where $z^{+}$and $z^{-}$correspond to the values of $q_{ \pm}$when $m$ goes from $m_{\min }$ to $m_{\max }$. If we now increase $L$ indefinitely, the integrals have value $\pi$, so

$$
P_{\mathrm{rad}}=\frac{4 \pi^{2} \sigma \mu^{2} L}{k_{\mathrm{cl}}} \sum_{m} J_{m}^{2}\left(k_{\mathrm{cl}} Q \sin \theta_{m}\right)\left(1+\cos ^{2} \theta_{m}\right)
$$

This expression is exactly the same as found in previous analyses (equation A17 of [4]) which neglected the rotation of the direction of polarization.

\section{Conclusions}

We have demonstrated that the equivalent current method can be employed to predict the bend loss in an optical fibre for both orthogonal polarizations of modal field. The same analysis is then carried out for a helically bent fibre and it is observed that rotation of polarization due to the torsion does not affect the radiation loss.

\section{Acknowledgements}

We thank Dr John D. Love of Australian National University for his inspiration and help. We also appreciate the comments and suggestions of the reviewers. This work is supported in part by NATO-AGARD, Guidance and Control Panel.

\section{References}

1. D. CHANG and E. F. KUESTER, IEEE J. Quantum Electron. 11 (1975) 903.

2. A W SNYDER and J. D. LOVE, Optical Waveguide Theory (Chapman and Hall, London, 1983).

3. J. D LOVE and A. W. SNYDER, Electron Lett. 23 (1987) 1109.

4. A ALTINTAŞ and J. D. LovE, Opt. Quantum Electron. 22 (1990) 213.

5. J. N ROSS, Opt. Quantum Electron. 16 (1984) 455.

6. S G TANYER, M.S. Thesis (Bilkent University, Ankara, Turkey, 1990) p. 25. 